\title{
Simple models for complex systems: exploiting the relationship between local and global densities
}

\author{
Mercedes Pascual • Manojit Roy • Karina Laneri
}

Received: 18 October 2010 / Accepted: 17 January 2011 / Published online: 11 March 2011

(C) The Author(s) 2011. This article is published with open access at Springerlink.com

\begin{abstract}
Simple temporal models that ignore the spatial nature of interactions and track only changes in mean quantities, such as global densities, are typically used under the unrealistic assumption that individuals are well mixed. These so-called mean-field models are often considered overly simplified, given the ample evidence for distributed interactions and spatial heterogeneity over broad ranges of scales. Here, we present one reason why such simple population models may work even when mass-action assumptions do not hold: spatial structure is present but it relates to global densities in a special way. With an individual-based predator-prey model that is spatial and stochastic, and whose mean-field counterpart is the classic LotkaVolterra model, we show that the global densities and
\end{abstract}

Electronic supplementary material The online version of this article (doi:10.1007/s12080-011-0116-2) contains supplementary material, which is available to authorized users.

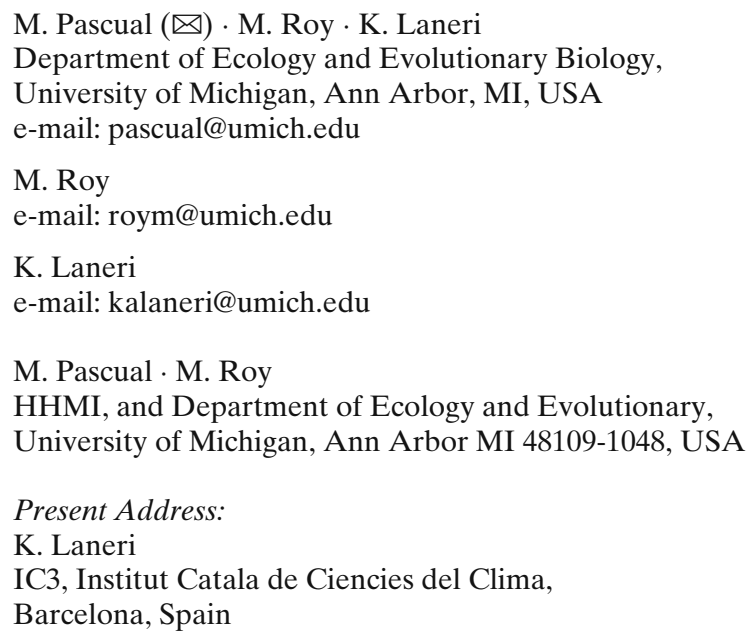

densities of pairs (or spatial covariances) establish a $b i$ power law at the stationary state and also in their transient approach to this state. This relationship implies that the dynamics of global densities can be written simply as a function of those densities alone without invoking pairs (or higher order moments). The exponents of the bi-power law for the predation rate exhibit a remarkable robustness to changes in model parameters. Evidence is presented for a connection of our findings to the existence of a critical phase transition in the dynamics of the spatial system. We discuss the application of similar modified mean-field equations to other ecological systems for which similar transitions have been described, both in models and empirical data.

Keywords From individuals to populations - Modified mean-field equations $\cdot$ Scaling $\cdot$ Implicit space in ecological models $\cdot$ Moment closure $\cdot$ Criticality

There are always scales and dimensions that are being ignored. And this is dangerous because the whole point is that all these sizes of turbulence are interconnected; they are both separate and continuous, feeding energy from large to small then back again-Gilles Foden; Turbulence, A novel of the atmosphere.

\section{Introduction}

In his 1992 Robert H. MacArthur Award Lecture titled 'The problem of pattern and scale in Ecology', Levin (1992) emphasized the central problem of linking dynamics across scales in ecology: in time, space and levels of organization. This problem lies at the 
heart of modeling complex systems, not only to understand their dynamics but also to identify the scales that determine their global behavior (Pascual 2005). In parallel with the development of models with increasing number of interacting components, such as individual- or agent-based models, a large number of theoretical studies in the past two decades have explored ways to simplify these high-dimensional formulations to capture their global, or aggregated, dynamics (e.g., Bolker and Pacala 1997; Keeling 1999; Filipe and Maule 2003; Pascual and Levin 1999a; Aparicio and Pascual 2007; Volz 2008). The resulting approximations can provide systems whose dynamics are easier to study and whose equations are easier to parameterize from empirical data. They can also help us understand how to incorporate implicitly the effects of variability at the smaller scales that are not explicitly represented in the model. This is particularly relevant because all ecological systems are nonlinear as a result of densityor frequency-dependent interactions, and nonlinearity allows variability to interact across scales, as illustrated by the paradigmatic example of fluid turbulence. Because variability typically occurs over a broad range of scales, any simple model must either choose to ignore the effects of unrepresented scales or incorporate these in some phenomenological way.

At one extreme of the spectrum, mean-field models aggregate all individuals into a single variable and ignore space completely, considering only global densities under the simplifying assumption that individuals are well mixed and they sample space at random in their interactions. We can ask whether such simple models belong mainly to textbooks and to the history of Ecology, together with their well-known ancestors by Lotka and Volterra (Lotka 1925; Volterra 1926), given that ecological systems are typically distributed, with interactions that are local in space or in other network topologies. However, mean-field models continue to find applications in a variety of problems and in particular those that involve confronting models with population-level data (e.g., Earn et al. 2000; Finkenstadt and Grenfell 2000; King et al. 2008). One approach that corrects the mean-field models to account for deviations from random mixing, and associated influences of spatial heterogeneity on the global dynamics of population densities, has relied on modifications of the functional forms describing interactions between individuals. An early example of this is found in the functional form proposed for the transmission rate in epidemiological models, which replaces a bilinear term (for the product of infected and susceptible individuals, $I S$ ) by a nonlinear term in which each density is raised to a power $\left(S^{\alpha_{1}} I^{\alpha_{2}}\right)$ (Severo 1969; Liu et al. 1987; Hochberg 1991; Gubbins and Gilligan 1997). A number of studies have relied on this type of functional form to formulate simple transmission models that were fitted to time series data (e.g., Bjornstad et al. 2002; Koelle and Pascual 2004; Koelle et al. 2005). There has been limited theoretical work to actually examine whether this functional form provides a good approximation to the aggregated, or global, population dynamics of systems with local interactions at the individual level. One exception concerns infectious disease dynamics with temporary immunity in a small-world network (Roy and Pascual 2006). Theoretical studies of individual-based predator-prey systems have also considered how the functional form of interaction rates at the aggregated population level are modified by local interactions, and noted modifications based on power functions of densities (Pascual and Levin 1999a; Roy et al. 2003).

A more extensively studied approach to simplify, or scale, spatial stochastic systems from individuals to populations has relied on incorporating the effects of third and higher order moments on the dynamics of pairs (e.g., Bolker and Pacala 1997; Keeling 1999; Sato and Iwasa 2000). Here, the equations describing the dynamics of global densities are modified to include terms that are a function of spatial variances and covariances (or "local" densities; Schlict and Iwasa 2006). The problem becomes one of suitably closing the system, since equations must be added for the dynamics of the second-order moments, but these include terms with higher order moments and so, in an infinite set of equations that relates variability across scales in an intimate fashion. Typically, the system is closed at the level of variances and covariances, by finding dependencies between moments (see Bolker and Pacala 1997 for an elegant example).

Here, we propose that for some spatial ecological systems these two simplification approaches, "modified mean-field equations" and moment-closure approximations, are related, and this is one reason why we may be able to use simple models that consider only mean, or global, densities, and ignore all other moments, even though these systems are highly structured in space and interactions are local, in a strong departure from the random mixing assumption. We rely on a spatial and stochastic predator-prey model where the individuals interact locally with near neighbors on a lattice; the mean-field counterpart of this model is the classic Lotka-Volterra predator-prey model with a carrying capacity in the growth of the prey. We show that the dynamics of the global predator and prey 
densities are well approximated by a modified meanfield model, whose functional forms incorporate power laws of these densities. This approximation appears to hold because the local densities (spatial covariances) in the system are themselves well approximated by functions of the global densities. Furthermore, these functions specifically involve power laws, not just in the long-term, or asymptotic, dynamics, but even earlier, in the transient regime as the system approaches the stationary state. In other words, as the spatial patterns self-organize in the system, the global and local densities establish a relationship that can be described by power laws. It follows that the global dynamics of the spatial system can be modeled as a function of mean densities alone, ignoring any higher order moments that would take into account spatial heterogeneities from neighborhood interactions. This is not because spatial variability does not matter; on the contrary it is fundamental. Spatial patterns arise in this system as the result of a critical phase transition, a percolation-type transition at which the system-wide connectivity of the prey changes dramatically, and power laws emerge in the size distribution of connected prey clusters. Similar types of transition and associated patterns have been described for a variety of spatial stochastic models in ecology, and scale-free patterns of this kind have been documented for different empirical systems, from vegetation in arid ecosystems to ant colonies to mussels in the intertidal (Pascual et al. 2002b; Guichard et al. 2003; Kefi et al. 2007; Scanlon et al. 2007; Sole 2007; Vandermeer et al. 2008; Kefi et al. 2011). We provide preliminary evidence for a connection between criticality in our predator-prey individual-based model and the robustness of the power-law exponents used to modify the predation rate in the modified meanfield equations. We speculate on the generality of these results to spatial dynamics that involve local birth and death processes that are density-dependent.

\section{The spatial stochastic model}

The model is implemented as an interacting particle system (IPS) (Durrett and Levin 1994). This type of stochastic model shares with the better known cellular automata, the discrete nature of space, here, a twodimensional grid of size $L^{2}$ in which individuals can occupy grid cells and interact locally with their neighbors. Each cell can be in one of three possible states: empty, occupied by a prey, or occupied by a predator. Because preys and predators rarely have an "activity range" encompassing the entire landscape, but have instead spatially restricted ranges for movement and interaction, we use the simplest activity neighborhoodthe four nearest neighbors of a focal cell. By contrast to discrete-time cellular automata, time is treated in an IPS as continuous. Transitions of a cell from one state to another are stochastic and their probabilities determined by a stochastic process in continuous time. The birth of prey onto empty space, the death of prey by predation, the corresponding birth of a predator, and the death of predators are all treated as Poisson processes, with their rates of determining their associated probabilities in a small interval of time. Moreover, the death of the predator is density-independent and a cell occupied by a predator becomes empty at a rate $\delta$. Predation rates depend instead on the number of predators in the local neighborhood of a prey. Thus, a cell that is occupied by a prey dies and becomes occupied by a predator at a rate that is proportional to the density of predators in its neighborhood, with constant of proportionality $\beta$. Finally, an empty cell becomes occupied by a prey also at a rate that depends on the local density of prey in its neighborhood, with constant of proportionality $\alpha$. We refer to this model as the "spatial" Lotka-Volterra model.

\section{The mean-field model and some approximations}

At the opposite end of the spatial model with local interactions, we can write the mean-field model obtained by assuming that individuals are well mixed and therefore, perceive local densities in their neighborhood that are equal to global ones. In other words, they sample space at random in the whole grid. Let $p, h, e(=1-p-h)$ denote the global/mean densities of prey, predator, and empty cells, respectively. The equations for this model are given by

$\mathrm{d} p / \mathrm{d} t=\alpha p e-\beta p h$,

$\mathrm{d} h / \mathrm{d} t=\beta p h-\delta h$,

where $\alpha, \beta$ and $\delta$ denote, respectively, (per capita) rates of prey growth, predation and predator death. These equations correspond to the well-known LotkaVolterra model with the growth of the prey limited by a carrying capacity. Both the processes of prey growth on empty space (at rate $\alpha p e$ ) and of predators hunting for prey (at rate $\beta p h$ ) are bilinear in the respective densities, which is the standard "mass-action" form characteristic of a well-mixed system. 
Model 1 can be simplified to a two-parameter model by rescaling the time by, for example, the parameter $\beta$ using the transformation $t^{\prime}=\beta t, \alpha^{\prime}=\alpha / \beta, \delta^{\prime}=\delta / \beta$, and then dropping the prime ' for convenience:

$\mathrm{d} p / \mathrm{d} t=\alpha p e-p h$,

$\mathrm{d} h / \mathrm{d} t=p h-\delta h$.

We use Eq. 2 as our mean-field (MF) LotkaVolterra model, with stable equilibrial densities given by

$p^{*}=\delta, \quad h^{*}=\frac{\alpha(1-\delta)}{1+\alpha} . \quad(0 \leq \delta \leq 1)$

We know that the MF model (Eq. 2) can only poorly capture the dynamics of global densities for the spatial model because interactions are local (see for example Fig. 2c, d), and that this will be the case for both the transients and asymptotic dynamics. The correct equations for the global densities of predator and prey can be easily written with pair-approximation approach familiar from a number of other studies (see for example Sato and Iwasa 2000). We let $[p h]$ and $[p e]$ denote the respective densities of prey-predator and prey-empty site pairs in the system. These correspond to spatial covariances (or local densities) as can be seen from writing $[p h]=\operatorname{cov}(p, h)+p h$, and $[p e]=\operatorname{cov}(p, e)+$ $p e$ (in the well-mixed MF model, covariances are zero and $[p h]=p h,[p e]=p e$ ). To emphasize the local nature of these pairs, we can also write $[p h]=p \rho_{h p}$ and $[p e]=e \rho_{p e}$, where $\rho_{h p}$ is the conditional probability of finding a predator in the local neighborhood of a prey, and similarly, $\rho_{p e}$ is the conditional probability of finding a prey in the local neighborhood of an empty site.

With this notation, we can write the exact equations for the spatial model as

$\mathrm{d} p / \mathrm{d} t=\alpha[p e]-[p h]$,

$\mathrm{d} h / \mathrm{d} t=[p h]-\delta h$.

As is typical from pair-approximation models, we see that the equations for global densities are not closed, and one would need a second set of equations for the pair densities (or second-order moments) $[p h]$ and [ $p e]$ in terms of triplets (or third-order moments) $[p p h]$, $[p h h],[p p e],[p e e]$ etc, and so on. One would then proceed to explore ways to close the system at the level of pairs (by, for example, assuming that triplet densities become simple functions of pairs), to approximate the dynamics of the spatial system with a model consisting of four equations, two for mean densities and two for spatial covariances.

This is not the path we explore here. We would instead like to use an even simpler approximation that writes the mean or global densities as a function of themselves alone and does not track covariances or higher order moments. This is in a sense what we do when we write simple temporal models at some aggregated level and ignore heterogeneities at smaller spatial scales. By considering Eq. 3, we see that this would work as long as the pair densities were themselves a function of mean densities. That is, we need $[p h]=f(p, h)$ and $[p e]=g(p, e)$, where $f$ and $g$ are yet unspecified functions. We can numerically extract these by simulating the spatial model and plotting $[p h]$ vs $p$ and $h$, to ask whether the function $f$ exists not just for the long-term dynamics but also for part of the transients, and what do these functions look like. Motivated by our earlier work (Pascual and Levin 1999a; Pascual et al. 2002a), we are especially interested in replacing bilinear functional forms with parametric nonlinearities, or "bi-power laws", such that $[p e]$ in Eq. 3 is replaced by $g(p, e)=c_{1} p^{a_{1}} e^{b_{1}}$, and $[p h]$ by $f(p, h)=c_{2} p^{a_{2}} h^{b_{2}}\left(a_{i}, b_{i}\right.$ and $\left.c_{i}>0, i=1,2\right)$. The MF Eq. 2 now take the modified form:

$\mathrm{d} p / \mathrm{d} t=\alpha c_{1} p^{a_{1}} e^{b_{1}}-c_{2} p^{a_{2}} h^{b_{2}}$,

$\mathrm{d} h / \mathrm{d} t=c_{2} p^{a_{2}} h^{b_{2}}-\delta h$.

We call Eq. 4 the "modified mean-field" (MMF) model, which, unlike the MF model (Eq. 2), can permit more complex solutions besides stable equilibria, including limit cycles (e.g., see Liu et al. 1987). The new parameters $c_{i}, a_{i}$ and $b_{i}(i=1,2)$ modify the respective functional forms of the prey's growth rate and the predation rate. In particular, $a_{i}$ and $b_{i}$ capture the nonlinear effects of deviations from random mixing caused by localized interactions. The original bilinear functional forms in the MF model are now nonlinear. For example, because $p, h$ are defined as densities, $a_{2}>$ 1 (assuming $b_{2}=1$ ) implies that a predator sees on average fewer prey in its neighborhood than in the wellmixed MF model. The overall per-capita predation rate is thus smaller than its corresponding value in the original model. Likewise, $0<a_{2}<1$ implies that a predator sees on average more prey in its neighborhood and that the per-capita predation rate is larger. Similarly, the value of the exponent $b_{2}$ influences the number of predators that a prey sees on average. Clustering of the prey is expected to reduce this rate as the prey are "protected" by neighboring conspecifics from interactions with predators. Finally, the coefficients $c_{i}$ capture only linear effects of the spatial patterns: they can decrease or increase (when $0<c_{i}<1$ and $c_{i}>1$, respectively) the overall rate but cannot modify its functional form. Such intuitive interpretations are possible one exponent (and coefficient) at a time, holding the others fixed 
(at their MF values). When all vary simultaneously, the interpretation of individual parameters becomes difficult but that of their combined effect on the overall rate is still possible.

\section{Results}

As a result of localized interactions, the spatial model develops spatial patterns characterized by clustering of the prey, empty and predator sites (Fig. 1a; compare with Fig. $1 \mathrm{~b}$ for the MF model at the same asymptotic prey density). Similar patterns of self-organization have been described in a number of stochastic spatial models and empirical data in ecology (Pascual et al. 2002b; Guichard et al. 2003; Roy et al. 2003; Kefi et al. 2007; Scanlon et al. 2007; Sole 2007; Vandermeer et al. 2008). The origin of these patterns in all these systems is to be found in the existence of a critical point, a particular value of the parameters (and corresponding density) at which the system exhibits a dramatic change in the connectedness of the clusters for one of the species (see Roy et al. 2003 for details in another predator-prey model). At this point, a giant cluster "percolates" through the lattice, spanning it from one end to the other. This reflects a critical phase transition, and it is at this critical or percolation point that system-wide spatial correlations develop in the grid, and correspondingly, power laws arise in many of the quantities characterizing the resulting spatial patterns. Because of these long-range spatial correlations, whose characteristic length extends beyond that of the interaction neighborhood, we expect the MF model with its assumption of random mixing to poorly approximate the global dynamics of densities. This correlation length is determined by the proximity of the system to the percolation point and is system-wide at this critical transition. Figure 1c shows for our predator-prey system, the probability of observing a percolating prey cluster that spans the grid from one end to the other, and that this quantity jumps from zero to one at a particular prey density $\bar{p}=0.54$ ( $\bar{p}$ denotes time-averaged asymptotic density). Figure $1 \mathrm{~d}$ shows that at this prey density, the size of prey clusters exhibits a power-law distribution. These patterns break down only progressively as we move away from the critical point towards subcritical prey densities, as has been described in related systems elsewhere (see Kefi et al. 2011 for a description of this in a number of models; and Roy et al. 2003 for more technical aspects). At these subcritical densities, longranged spatial correlations are still present and cluster sizes scale approximately as a power law but over a restricted range of sizes, but there are no spanning clusters. On the other side of the critical point, at supercritical densities, the power-law pattern degenerates at a faster pace, and what remain are mostly a large
Fig. 1 Spatial patterns at the stationary state in the spatial and mean-field models at $\bar{p}=0.5(\mathbf{a}, \mathbf{b})$. The prey, predator, and empty sites are drawn in orange, white, and black pixels, respectively. In (c), the percolation probability $P$ is plotted as a function of prey density $\bar{p}$, which indicates a percolation point at $\bar{p}=p_{c}=0.54$ (see text for details). As expected at this critical transition, the size distribution of prey clusters is a power law. This distribution is shown in (d) for the critical density $\bar{p}=0.54$ on $\log -\log$ scale (see text)

\section{a}
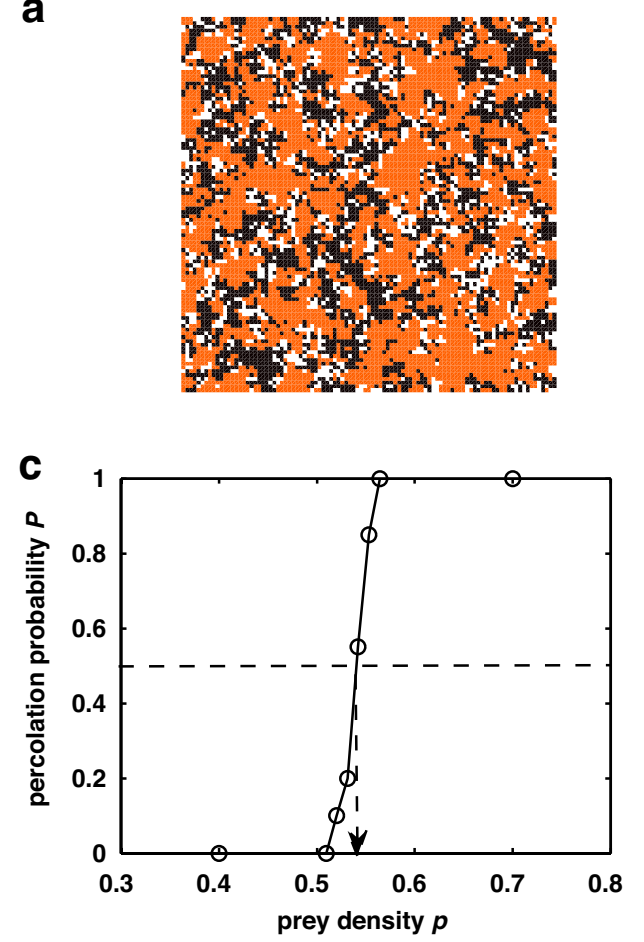

b
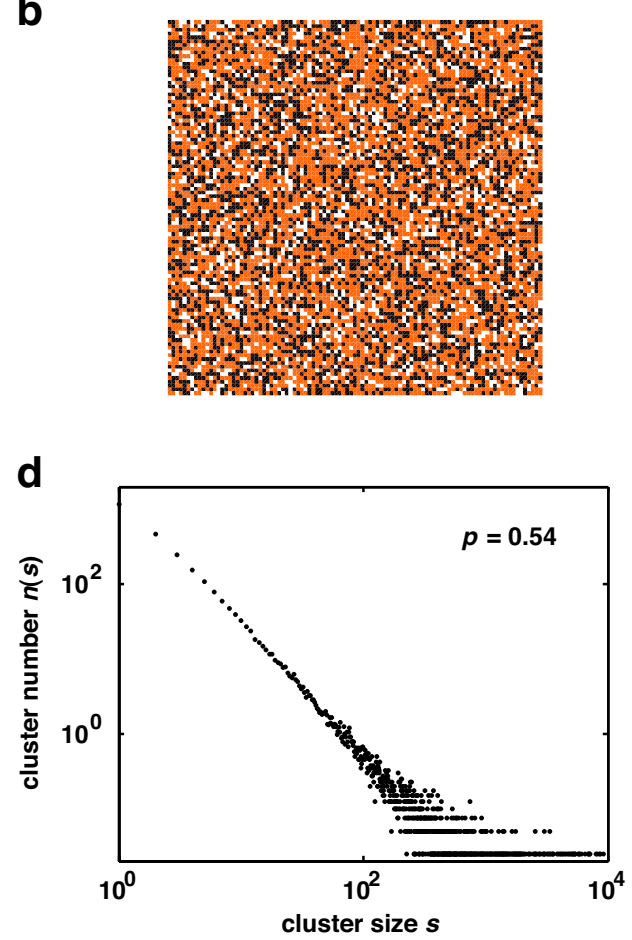
spanning cluster and many small ones. Note that, in the $(\alpha, \delta)$ parameter space used in the plots of Figs. 2 and 3 below, the percolation point becomes a line, as the critical prey density $\bar{p}=0.54$ can be obtained by appropriately varying both $\alpha$ and $\delta$.

As expected, because of such highly non-random and aggregated spatial patterns, the MF model is unable to capture the temporal dynamics of total densities in the spatial model (Fig. 2c, d: compare the time series in red solid vs. black dashed lines). This is not surprising as no large spatial clusters can arise from randomized interactions in the well-mixed system (see Fig. 1b). Our objective here is to explore the degree to which the MMF model (Eq. 4) can improve upon such MF predictions in capturing the outcome of the spatial model,

a
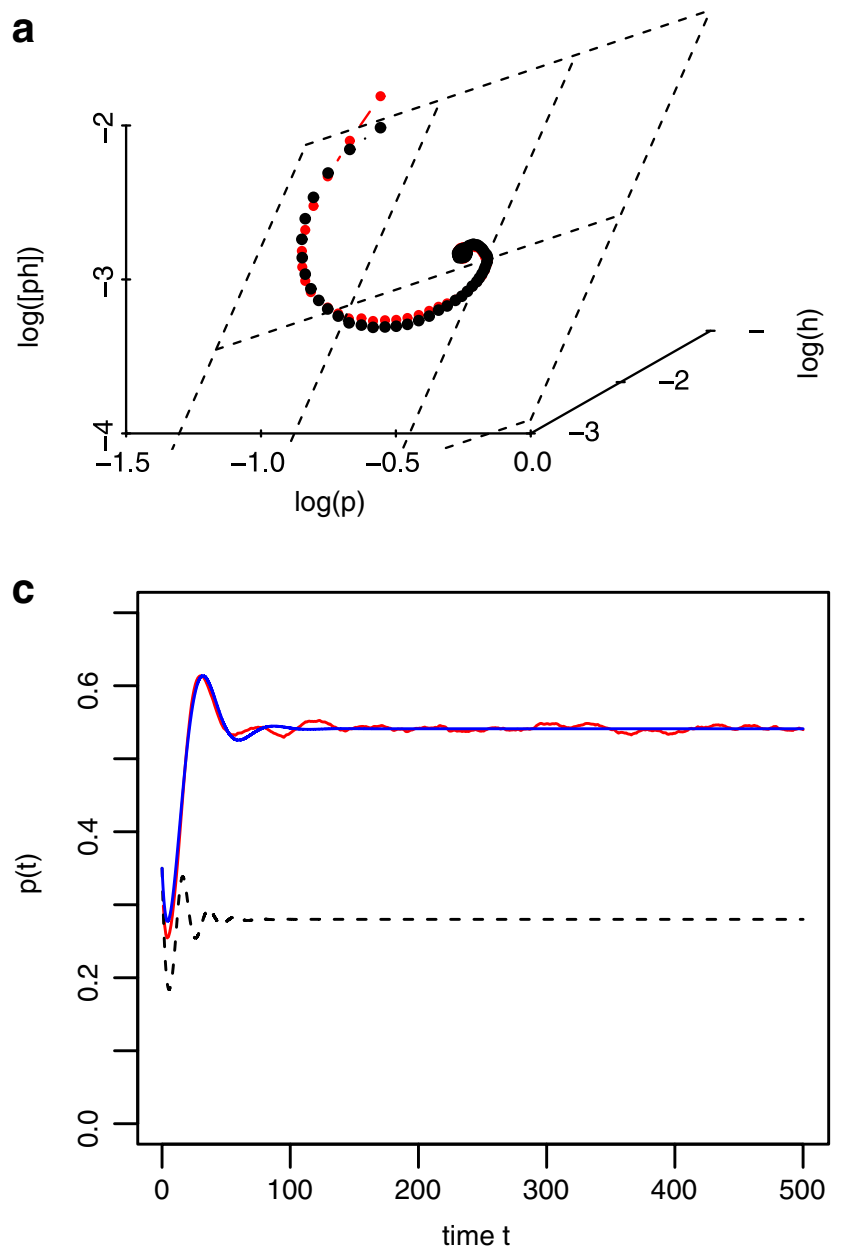

Fig. 2 a, b Trajectory plots for $p-h-[p h]$ and $p-e-[p e]$ as a function of time on a three-dimensional log scale (red dots) together with their corresponding regression planes and projections on this plane (black dots) (see text). The model parameters $\alpha, \delta$ are chosen so that the trajectory settles onto the critical stationary state $\bar{p}=0.54$. The fitted parameters for the planes and corresponding MMF coefficients are $\left(a_{1}, b_{1}, c_{1}, a_{2}, b_{2}, c_{2}\right)=$ $(0.2,0.59,0.19,0.59,1,0.4) . R^{2}$ values for the fits in $(\mathbf{a}, \mathbf{b})$ are both in the stationary and transient regimes. We return later to the role of criticality, and ask whether powerlaw scalings in the spatial patterns (such as Fig. 1d) can influence our findings on scaling the temporal dynamics of the system from individuals to global population densities, as in Eq. 4.

We begin by simply plotting for a particular set of parameters $\alpha$ and $\delta$, the trajectory in time of the pair density $[p h](t)$ as a function of the corresponding global densities $p(t)$ and $h(t)$, in a three-dimensional $\log$ space. Figure 2a illustrates that this trajectory, in red, lies approximately on a plane (see also Fig. S1 in the Electronic supplementary material). Interestingly this means that the local covariances for predator and prey (or local densities) are a smooth function
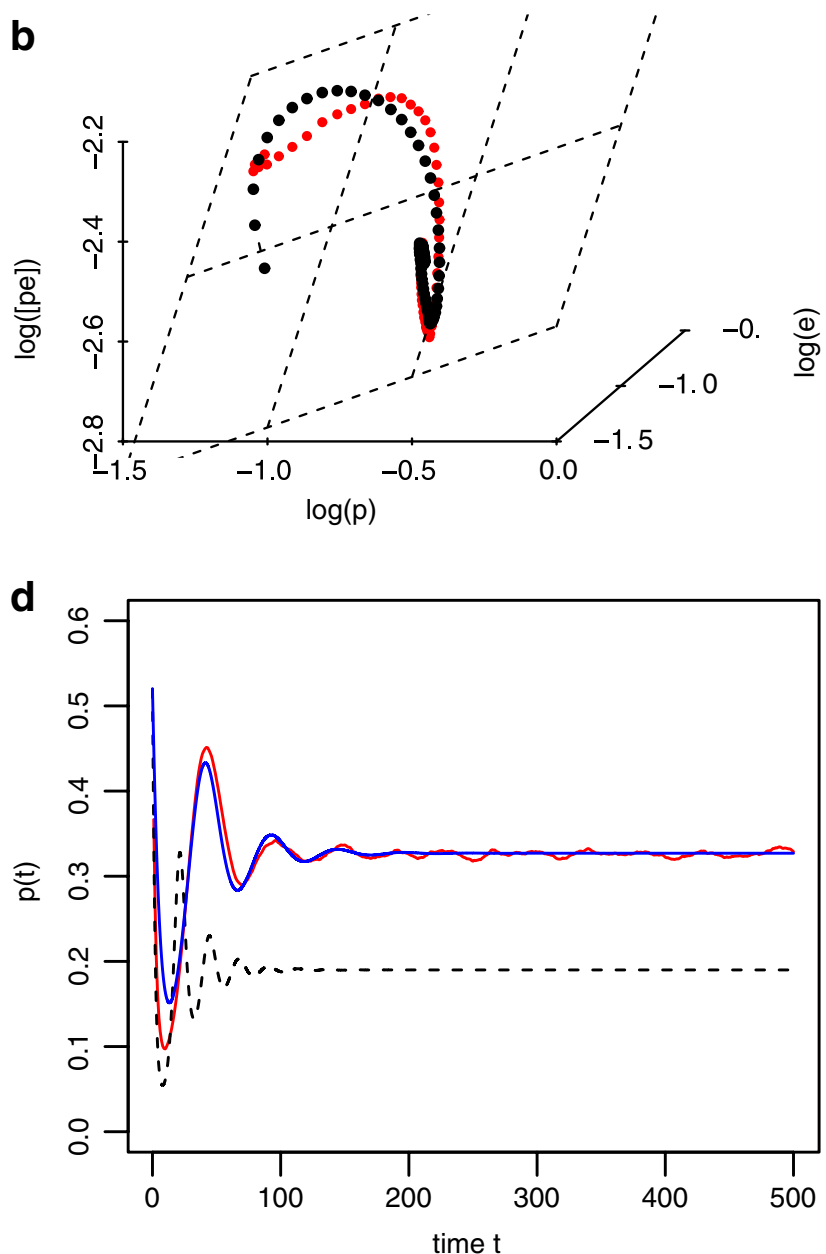

0.99 and 0.76 , respectively. c The corresponding prey time-series data $p(t)$ of the spatial model (in red) are compared with the solutions of the MF model (Eq. 2) (black dashed line) and MMF model (Eq. 4) (in blue). d A similar comparison is shown for another trajectory that corresponds to a subcritical density $\bar{p}=$ 0.3 , and the fitted MMF coefficients are $\left(a_{1}, b_{1}, c_{1}, a_{2}, b_{2}, c_{2}\right)=$ $(0.59,0.4,0.19,0.67,0.9,0.34)$. These values do not differ much from those for the critical trajectory in (c) 

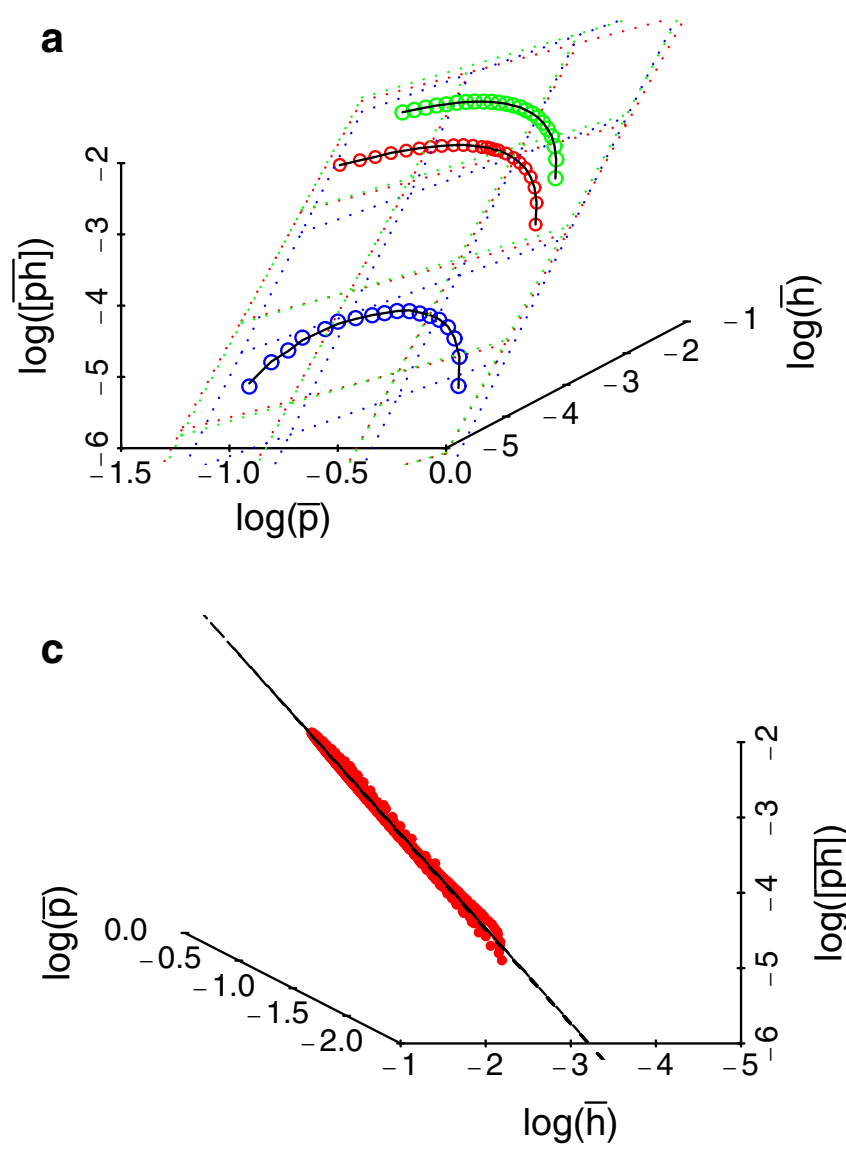

Fig. 3 The points $\bar{p}-\bar{h}-\overline{[p h]}$ and $\bar{p}-\bar{e}-\overline{[p e]}$ at the stationary state are plotted together with their regression planes on a $3 \mathrm{D} \log$ scale, for three fixed values of $\alpha=0.1$ (in blue), 0.5 (in red), and 0.9 (in green), while $\delta$ is varied (see text for details) (a, b). Similar plots for these points at the stationary state (in red) are

of global densities, and that this function can be approximated well by a bi-power law (because on a threedimensional log space bi-power laws describe a plane; see below). This holds for a substantial part of the transients: after some initial time, the trajectory appears to already settle on this plane. Thus, we can use this trajectory to fit the exponents $a_{2}, b_{2}$ and coefficient $c_{2}$ of the bi-power law function $c_{2} p^{a_{2}} h^{b_{2}}$ in the MMF model (Eq. 4). A similar pattern holds for the [pe] pair function $c_{1} p^{a_{1}} e^{b_{1}}$ (Fig. 2b), although the trajectory here exhibits larger deviations from a plane (a feature that recurs throughout our results below and which we elaborate in the "Discussion"). We compute the MMF parameters, $c_{1}, a_{1}, b_{1}, c_{2}, a_{2}, b_{2}$, by such logregression fits; on log axes, the regression model $y=$ $c_{2} p^{a_{2}} h^{b_{2}}$ describes a regression plane $\log (y)=\log \left(c_{2}\right)+$ $a_{2} \log (p)+b_{2} \log (h)$ with intercept $\log \left(c_{2}\right)$ on the $y$-axis (and similarly for $y=c_{1} p^{a_{1}} e^{b_{1}}$ ). Figure $2 \mathrm{c}$, d show that the resulting MMF model provides a much better ap-
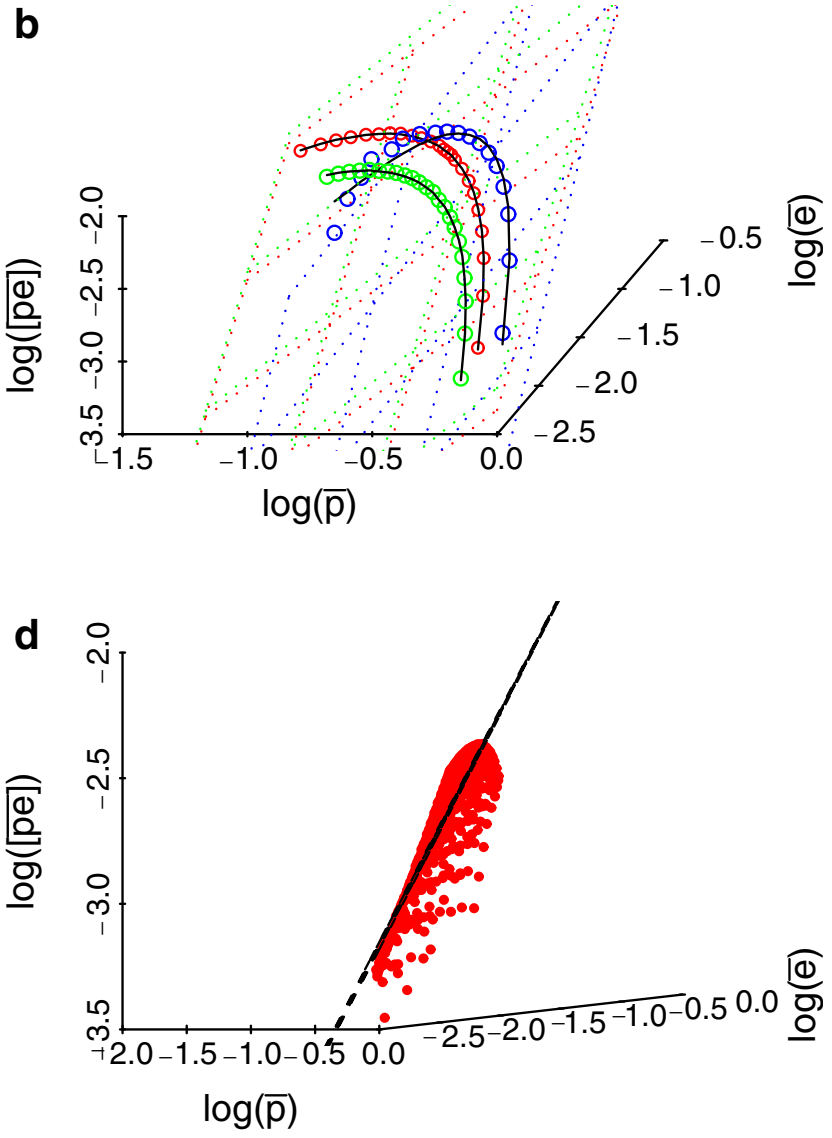

shown when both $\alpha$ and $\delta$ are simultaneously varied (see text), along with their regression planes, oriented so that each plane is viewed along its edge $(\mathbf{c}, \mathbf{d}) . R^{2}$ values for the fits in $(\mathbf{c}, \mathbf{d})$ are 0.99 and 0.76 , respectively

proximation to the dynamics of the spatial system over a large range of densities, not just for the equilibrium but also for part of the transients. Similar results were obtained for other values of $\alpha$ and $\delta$ (not shown here, but see discussion on robustness below). These findings provide a justification for the type of functional form in the MMF model, where the "modified" functional form for the respective rates of predation and prey growth incorporate the densities to a power. (Note that the approach taken here to estimate the parameters of the modified functional forms fundamentally differs from fitting the MMF model to the temporal trajectories of the global prey and predator densities in the spatial system. Given the number of parameters and number of differential equations, one could always trivially fit the MMF model to these transients. We considered instead the relationship between second and first-order moments to fit the exponents, and then examined how well the MMF model that includes the resulting 
functional forms approximates the aggregated dynamics of the spatial system).

We ask next whether the exponents in these modified functional forms are in some way robust to changes in the parameters of the spatial model. In other words, do we need a different correction to the bilinear functional forms of the mean-field model, or can we find a set of exponents that is independent and "works" across parameters $\alpha$ and $\delta$ ? To address this question, we no longer consider the trajectories of pair densities but their values at the stationary state, and vary the parameter $\delta$ first (for a given $\alpha$ ), to obtain a number of time-averaged asymptotic points $\overline{[p h]}$ vs. $\bar{p}$ and $\bar{h}$ on the same three-dimensional log plot. Figure 3 a shows that these points lie exactly on the same plane. Thus, at the stationary state, the pair densities are again a bi-power law function of the global densities, and a plane fits the points extremely well. A single set of exponents $a_{2}, b_{2}$ and intercept $c_{2}$ describe this relationship for a range of values of the dynamical parameter $\delta$ in the spatial model; and the same pattern is observed for exponents $a_{1}, b_{1}$ and intercept $c_{1}$ for the stationary $\overline{[p e]}$ pairs, although the fit is less impressive (Fig. 3b). We also observe similar relationships when we vary $\alpha$ keeping $\delta$ fixed (not shown).

We can go further and consider the full range of values of $\delta$ and $\alpha$, and vary both parameters simultaneously, to obtain a family of points in our threedimensional space of pair densities versus global densities from the stationary state of each numerical simulation of the spatial model. Figure $3 \mathrm{c}$ shows a remarkable pattern for the density of predator-prey pairs as a function of predator and prey densities: the whole cloud of points lies very close to a plane. This means that a single bi-power law, and correspondingly a single set of exponents and intercept, characterizes the relationship between this second-order moment and the first-order moments in this system at the stationary state, as we vary the dynamical parameters. It follows that a single bi-power law can be used to "modify" the predation rate of the original mean-field model. A similar analysis for the prey-empty pairs shows more scatter in their relationship with the global densities for the prey and empty sites (Fig. 3d). Here, a plane does not fit as well the whole set of points, and there is more variation in the values of the exponents for the different dynamical parameters.

We are interested in the possible connection between the existence of a critical phase transition in the system, at the percolation point described above, and the robustness of the exponents' values for the predator-prey pair. This is motivated by the observation that characteristic patterns of the prey clusters, and in particular of the boundary of these clusters at which predator-prey interactions take place, arise at this critical point. The relevance of the critical point $p_{c}(=0.54)$ can be investigated by examining whether the exponents obtained in its proximity best capture the relationship between the density of pairs and the global densities, everywhere in parameter space. In other words, does the plane defined by parameters in the proximity of the critical transition, provide a better fit to this relationship than that defined by parameters further away from it? To address this question, we consider subsets of points from Fig. 3c, d corresponding to different ranges of prey densities, and therefore, to different distances from the critical point $p_{c}$ (in Fig. 1c). We subdivide the set of data points $p-h$ - $[p h]$ and $p$ $e$-[pe] of Fig. 3c, d, which span the range $0.1 \leq \bar{p} \leq$ 0.9 , into smaller density intervals $\left[\bar{p}_{i}, \bar{p}_{j}\right]$, and carry out separate log-regression fits of the subset of data within each interval. (These intervals are chosen such that all of them have similar number of data points, in order to avoid any potential bias in the quality of fit). We then compute the "distance" $d$ between the entire set of points (in Fig. 3c, d) and the regression plane for the subset of data belonging to each of the above prey-density intervals. This distance is computed as the length of the normal vector drawn from each point to the plane, averaged over all points. Figure 4a, b show these plots versus the intervals, and the "critical interval" $[0.5,0.6]$ (that contains the critical point $\left.p_{c}=0.54\right)$ is shown by a short vertical arrow in each plot. The distance decreases rapidly as we approach the critical point from above, that is, for planes defined by supercritical dynamics. There is then a broad region where the distance exhibits a minimum.

Supplementary Fig. S2 shows the values of the exponents $a_{1}, b_{1}, a_{2}, b_{2}$ resulting from the individual regressions for each density interval. The MF value $a_{1}=$ $b_{1}=a_{2}=b_{2}=1$ is indicated by a horizontal dashed line, and the critical interval $[0.5,0.6]$ is marked again by a short vertical arrow. The exponents (and intercept, not shown) significantly differ from their MF value over much of the plot range, with the exception of $a_{2}$. The robustness of the exponents observed in Fig. 3c is reflected here in the little variation of $b_{2}$ and also of $a_{2}$ in the subcritical region (except for low prey densities). Values of $a_{2}$ close to one and $b_{2}$ larger than one tell us that a prey sees on average fewer predators than in the well mixed system. In combination with $c_{2}<1$, this leads to a reduced overall predation rate as the results of the prey's patchiness. Moreover, the average per-capita predation rate varies nonlinearly with the predator's density, increasing more rapidly as this density becomes larger. 


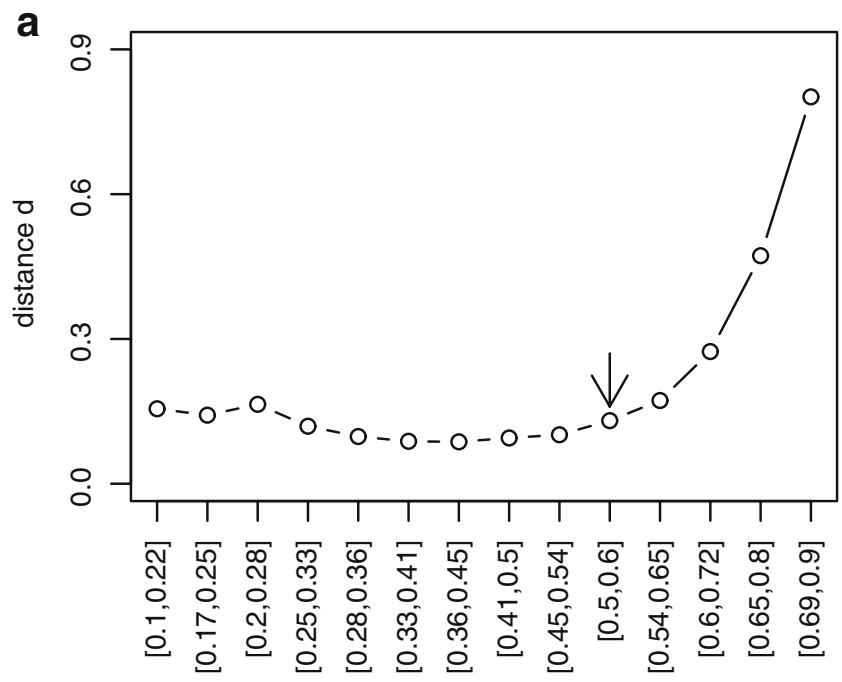

Fig. 4 The two plots $(\mathbf{a}, \mathbf{b})$ show the average distance between the data points in Fig. 3c, d, respectively, and the regression plane for each density interval $\left[\bar{p}_{i}, \bar{p}_{j}\right]$, where the plane is described by the fitted MMF coefficients $a_{2}, b_{2}, c_{2}$ and $a_{1}, b_{1}, c_{1}$ computed for

Finally, the MMF system can be used to examine the overall effect of local spatial structure on the dynamics of global densities. The main effect of space in our stochastic system is that of modifying the global density of the species at the steady-state: that is, the spatial system always exhibits a higher density of prey

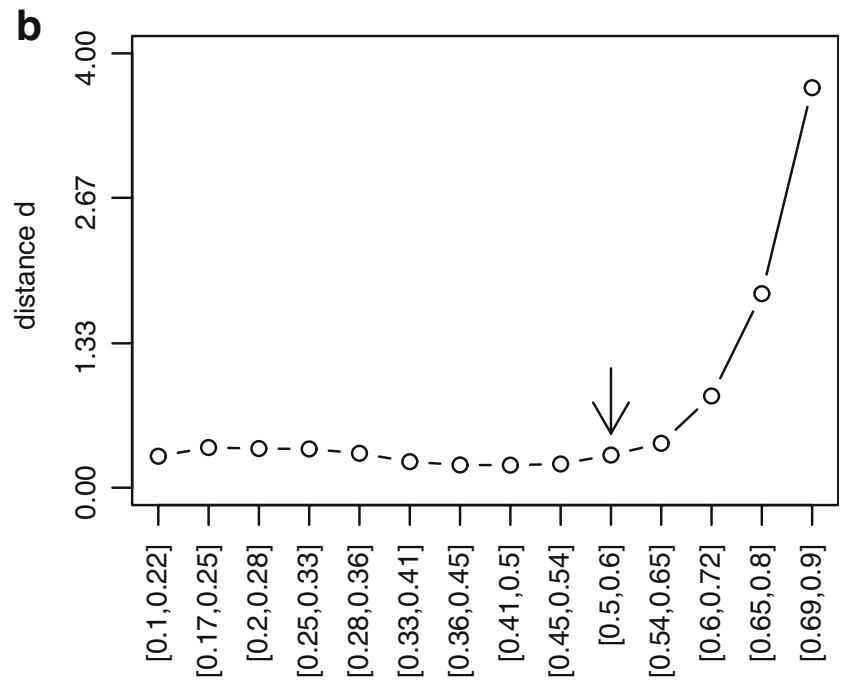

each interval (e.g., see Fig. S2 in the Electronic supplementary material and text); the averaging is done over all data points. The vertical arrow again shows the critical interval $[0.5,0.6]$

and correspondingly, a lower density of predators, than the MF model, and this is also the case for the MMF approximation. Figure 5 illustrates this with a comparison of the steady-state prey densities for the three models (MMF, MF, and spatial) for different values of the parameter $\delta$. Local interactions effectively
Fig. 5 Steady-state prey density versus the predator's mortality rate $\delta$ for the three different models: spatial (solid red), MMF (solid blue), and MF (broken black line) (see text for details)

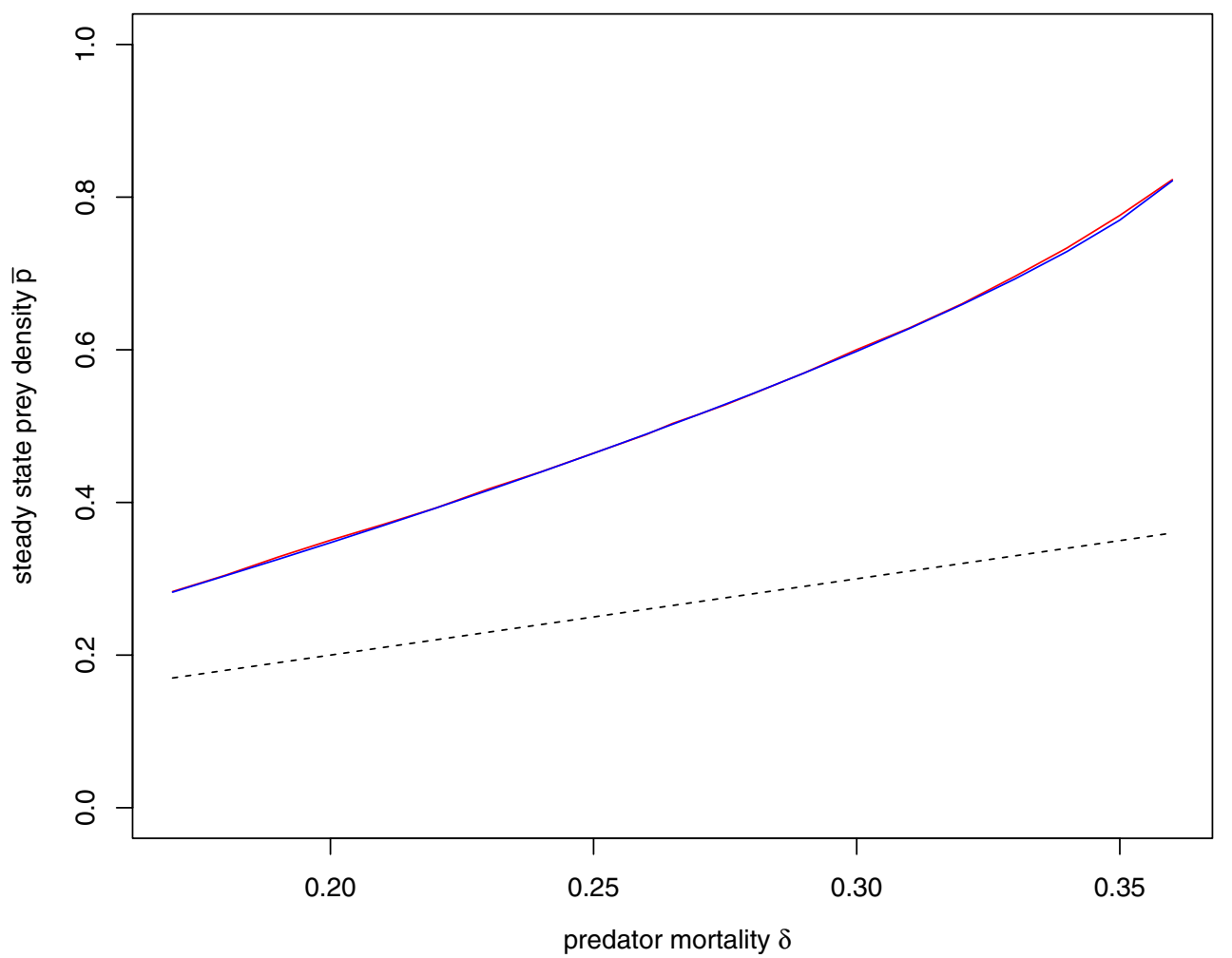


decrease the predation rate, and therefore, the predator's growth rate.

\section{Discussion}

Simple temporal models that ignore the spatial nature of interactions and track only the changes in mean quantities (such as global densities), are typically used under the unrealistic assumption that individuals are well-mixed. These so-called mean-field models are often considered overly simplified given the ample evidence for distributed interactions and spatial heterogeneity over broad ranges of scales. There is ample literature on the clear trade-offs in the respective advantages and disadvantages of simple vs. complex models, such as individual-based models that track interactions in space or other sorts of networks (e.g., DeAngelis and Gross 1992; Levin 1992). Our results illustrate, with a toy model that tracks individuals, that there is another reason why population models that only consider global densities may be applicable, not because mass-action assumptions apply and spatial structure is absent, but because the spatial structure relates to global densities in a special way.

We have shown that in our individual-based predatorprey model, the spatial covariances (or local densities) that develop as a result of local interactions establish a relationship with the global population densities. Interestingly, they do so not only in the stationary states, but even in the transient dynamics. Thus, even though the system has not yet settled onto its stationary spatial patterns, it has sufficiently self-organized to exhibit a relationship between the first- and second-order moments of the spatial distribution. As the spatiotemporal dynamics continue to evolve, this relationship persists both in time, after an initial phase of the transients, for a particular set of dynamical parameters, and also at the stationary state, when these parameters are simultaneously varied. Moreover, this relationship can be approximated by a bi-power law, so that in our system we can effectively replace the bilinear functional form of the predation rate in the classic LotkaVolterra equations by a bi-power law, where each of the prey and predator mean densities is raised to a power. Such modification has been proposed before for the transmission rate in epidemiological models (Severo 1969; Liu et al. 1987; Hochberg 1991; Gubbins and Gilligan 1997) as a phenomenological correction to the mean-field description. Here, the basis for this modification emerges from the individual-based dynamics itself. A similar relationship between local and global densities was exploited for disease transmission in a social network (Roy and Pascual 2006), with a focus however on the topological properties of the network and not on the robustness of exponents to parameter variation.

Simplifications of ecological models based on moment closure techniques have been used in a variety of systems (e.g. Bolker and Pacala 1997; Keeling 1999; Pascual and Levin 1999b, Sato and Iwasa 2000). However, the solution to the infinite hierarchy of equations that develops from this approach has been to close the system at the level of the second-order moments, with the resulting system tracking the temporal dynamics of mean quantities as well as variances and covariances. Our results suggest that we may be able to close some systems at the level of the means, and model the means only as a function of themselves, because the variances and covariances have approximate functional relationships with these means. This provides a justification for the use of modified mean-field models in which the effects of spatial variability at unresolved (smaller) spatial scales is not ignored, but instead represented implicitly via a modification of the functional form representing interactions at these scales.

One limitation of this approach is that we do not know a priori the appropriate functional form and we did not derive it here from first principles. Thus, the exponents themselves were not mechanistically related to the individual-level parameters of interactions. This is a problem when our objective is to simplify a detailed model that we have successfully parameterized. However, this is not always the case; we often recognize the potential complexity of the systems we would like to model but are unable to parameterize the models themselves, and would like to write a simpler model that captures in some way the underlying variability we have neglected. Theoretical investigations of this sort can suggest the form of such simpler models and give us a basis to consider for their use, especially in cases where the parameters of the models will be obtained at the aggregated level. They can also suggest specific patterns to test empirically, for example, to examine the relationship over time between first- and second-order moments.

Many open questions remain. Do similar results apply to other systems with birth and death processes? It would be interesting to consider individual-based models whose mean-field counterparts have functional forms that are not bilinear (such as those with type II or type III functional forms in the predation term). Both moment closure and MMF formulations can help us examine the effect of local spatial structure on the dynamics of global densities. Here, this effect is that of modifying steady-state densities (Fig. 5). For nonlinear 
functional forms, spatial structure may also change the long-term qualitative dynamics, by transforming a limit cycle into decaying oscillations approaching an equilibrium (Pascual and Levin 1999a). It is an open question whether the bifurcation analysis of a MMF model will show qualitative changes in dynamics consistent with those of the spatial stochastic system. The case of seasonally-forced dynamics would be especially interesting in this respect.

Moreover, what are the key features of systems where modified mean-field models with functional modifications including power laws apply? Can we relate the exponents of the modified functional form to a specific geometrical/structural feature of the spatial patterns? This would be of practical use. In particular, we can conjecture that the modifications in some way relate to the fractal dimension of the boundaries of the clusters where the interactions actually take place. Models for consumer-resource interactions between plants and herbivores have been proposed that explicitly consider the fractal dimension of resources (Ritchie 2009); the resulting functional forms also include exponents. In this approach, however, the spatial pattern of the resource is given; in our model, it emerges from the dynamics. It would be interesting to consider the possible relationship between these approaches.

Finally, we presented a few results that suggest a connection between the described relationship between moments and the existence of a percolation-type critical phase transition in the system. We have also shown a somewhat surprising robustness of this relationship for the predator-prey covariances: the plane that describes approximately their relationship to global prey and predator densities is largely invariant with changes in the dynamical parameters of the individual-based model. This was not the case, however, for the other covariance between prey and empty sites. Why is this so? Our conjecture is that for the former, the two relevant dynamical processes, the growth of the prey and the growth of the predator (replacement of the prey through predation) are both birth/death processes that depend on local densities. By contrast, the growth of empty sites is a point process that does not depend on the state of the local neighborhood. More generally, it is evident that criticality in this system is important to the emergence of the spatial patterns, especially their power-law scalings. It remains an open question how it relates to the scaling or simplification of the dynamics we have described here. This is relevant beyond theoretical considerations to the kinds of systems for which this type of simplification would apply. A number of ecological systems in which birth and death processes are locally density-dependent have been shown to ex- hibit similar patterns of self-organization, characterized by cluster size distributions that closely resemble power laws in models and in empirical data (Guichard et al. 2003; Kefi et al. 2007; Scanlon et al. 2007; Sole 2007; Vandermeer et al. 2008). The models all exhibit a percolation type transition, and this suggests that a critical phase transition of this kind underlies the observation of the corresponding power law like patterns in nature. The previously described relative robustness of these patterns to changes in parameters, as one moves away from their particular values at the critical point, would make their empirical observation possible (Pascual et al. 2002b; Roy et al. 2003; Kefi et al. 2011). We conjecture that modified mean-field equations such as the ones presented here will be of relevance to this family of ecological systems.

Acknowledgements We thank Simon Levin for the "inspiration" and the many interesting conversations throughout the years on problems of scale. We are also grateful to Alain Franc for early discussions on this work and to the James S. McDonnell Foundation for the support (to M.P.). M.P. is an investigator of the Howard Hughes Medical Institute.

Open Access This article is distributed under the terms of the Creative Commons Attribution Noncommercial License which permits any noncommercial use, distribution, and reproduction in any medium, provided the original author(s) and source are credited.

\section{References}

Aparicio J, Pascual M (2007) Building epidemiological models from r0: an implicit treatment of transmission in networks. Proc R Soc Lond B 274:505-512

Bjornstad ON, Finnkenstadt BF, Grenfell BT (2002) Dynamics of measles epidemics: estimating scaling of transmission rates usning a time series sir model. Ecol Monogr 72:169-184

Bolker B, Pacala S (1997) Using moment equations to understand stochastically driven spatial pattern formation in ecological systems. Theor Popul Biol 52:179-197

DeAneglis D, Gross LJ (eds) (1992) Individual-based models and approaches in ecology. Chapman \& Hall, New York

Durrett R, Levin S (1994) On the importance of being discrete (and spatial). Theor Popul Biol 46:363-394

Earn D, Rohani P, Bolker B, Grenfell B (2000) A simple model for complex dynamical transitions in epidemics. Science 287:667-670

Filipe J, Maule M (2003) Analytical methods for predicting the behaviour of population models. Math Biosci 183:15-35

Finkenstadt B, Grenfell B (2000) Time series modelling of childhood diseases: a dynamical system approach. Appl Stat 49:187-205

Gubbins S, Gilligan C (1997) A test of heterogeneous mixing as a mechanism for ecological persistence in a disturbed environment. Proc R Soc Lond B 264:227-232

Guichard F, Halpin P, Allison G, Lubchenco J, Menge B (2003) Mussel disturbance dynamics: signatures of oceanographic forcing from local interactions. Am Nat 161:889-904 
Hochberg M (1991) Non-linear transmission rates and the dynamics of infectious disease. J Theor Biol 153:301-321

Keeling M (1999) Correlation equations for endemic diseases. Proc R Soc Lond B Biol Sci 266:953-961

Kefi S, Rietkerk M, Alados C, Peuyo Y, Papanastasis V, ElAich A, de Ruiter P (2007) Spatial vegetation patters and imminent desertification in mediterranean arid ecosystems. Nature 449:213-217

Kefi S, Rietkerk M, Roy M, P de Ruiter AF, Pascual M (2011) Robust scaling in ecosystems and the meltdown of patch size distributions before extinction. Ecol Lett 14:29-35

King A, Ionides E, Pascual M, Bouma M (2008) Inapparent infections and cholera dynamics. Nature 454:877-880

Koelle K, Pascual M (2004) Disentangling extrinsic from intrinsic factors in disease dynamics: a nonlinear time series approach with an application to cholera. Am Nat 163:901-913

Koelle K, Rodo X, Pascual M, Yunus M, Mostafa G (2005) Refractory periods to climate forcing in cholera dynamics. Nature 436:696-700

Levin S (1992) The problem of pattern and scale in ecology: the robert h. macarthur award lecture. Ecology 73:1943-1967

Liu W, Hethcote H, Levin S (1987) Dynamical behavior of epidemiological models with non-linear incidence rates. J Math Biol 25:359-380

Lotka A (1925) Elements of physical biology. Williams and Wilkins, Baltimore

Pascual M (2005) Computational ecology: from the complex to the simple and back. PloS Comp Biol 1:101-105

Pascual M, Levin S (1999a) From individuals to population densities: searching for the intermediate scale of nontrivial determinism. Ecology 80:2225-2236

Pascual M, Levin S (1999b) Spatial scaling in a benthic population model with density-dependent disturbance. Theor Popul Biol 56:106-122
Pascual M, Roy M, Franc A (2002a) Simple temporal models for ecological systems with complex spatial patterns. Ecol Lett 5:412-419

Pascual M, Roy M, Guichard F, Flierl G (2002b) Cluster size distributions: signatures of self-organization in spatial ecologies. Phil Trans R Soc Lond B 357:657-666

Ritchie M (2009) Scale, heterogeneity, and the structure and diversity of ecological communities. Princeton University Press, Princeton

Roy M, Pascual M (2006) On representing network heterogeneities in the incidence rate of simple epidemic models. Ecol Complex 3:80-90

Roy M, Pascual M, Franc A (2003) Broad scaling region in a spatial ecological system. Complexity 8:19-27

Sato K, Iwasa Y (2000) Pair approximation for lattice-based ecological models. In: Dieckmann U, Law R, Metz J (eds) The geometry of ecological interactions. Cambridge University Press, Cambridge, pp 341-358

Scanlon T, Caylor K, Levin S, Rodriguez-Iturbe I (2007) Positive feedbacks promote power-law clustering of kalahari vegetation. Nature 449:209-213

Schlict R, Iwasa Y (2006) Deviation from power-law, spatial data of forest canopy gaps, and three lattice models. Ecol Model 198:399-408

Severo N (1969) Generalizations of some stochastic epidemic models. Math Biosci 4:395-402

Sole R (2007) Scaling laws in the drier. Nature 449:151-153

Vandermeer J, Perfecto I, Philpott S (2008) Clusters of ant colonies and robust criticality in a tropical agroecosystem. Nature 451:457-459

Volterra V (1926) Fluctuations in the abundance of species, considered mathematically. Nature 118:558-560

Volz E (2008) Sir dynamics in random networks with heterogeneous connectivity. J Math Biol 56:293-310 